\title{
Presentation of Two Cases Diagnosed with Brucella Endocarditis
}

\author{
Şükran Köse, Süheyla Serin Senger, Gürsel Ersan, Filiz Oğuz and Lütfiye Kuzucu
}

\begin{abstract}
Brucellosis is an important disease in developing countries. Endocarditis is a complication of brucellosis with the highest mortality. Although the most generally accepted therapy is the combination of medication and surgery, it has been reported that antibiotic treatment only might also be adequate. We present two cases for whom antibiotic treatments were initiated, and a follow-up surgery was planned for one of them. The surgery could not be done due to death of the patient, but the other patient fully recovered with antibiotic treatment only. Optimum treatment and prognostic criteria are not well defined for brucella endocarditis, but medical therapy alone can be considered for some chosen patients.
\end{abstract}

Key words: brucellosis, brucella endocarditis, zoonotic infections

(Intern Med 51: 953-955, 2012)

(DOI: 10.2169/internalmedicine.51.7103)

\section{Introduction}

Brucellosis is a zoonotic infection that is endemic in Mediterranean and Middle East regions. It effects many systems and appears with different findings and symptoms. Endocarditis is a complication of brucellosis with the highest mortality. Brucella related endocarditis has been reported to be less than $1 \%$ of all brucellosis cases in some studies (1). The aortic valve is the most commonly affected region in Brucella endocarditis. The disease rarely involves mitral valve. Treatment of Brucella endocarditis is still a disputable issue (2). Although treatment is controversial, the most widely accepted practice is combination of medical and surgical therapies. While it has been mostly suggested to remove the infected valves and replace them in addition to aggressive antibiotic treatment, it has been reported that only antibiotic treatment might also be adequate (3).

In this report we present two cases of Brucella endocarditis. The patients were examined by transthoracic echocardiography (TTE) with the definitive clinical diagnosis made in accordance with Modified Duke criteria for the diagnosis of infective endocarditis (4). For one of the patients surgery was planned following an initial medical therapy, whereas for the other patient only medical therapy was chosen due to a stable hemodynamic condition, lack of symptoms, and mild cardiac involvement. The patients had different outcomes even though their initial treatments were the same.

\section{Case Report}

\section{Case 1}

A 35-year-old man was admitted to hospital due to fever and weight loss. The patient had been diagnosed with chronic obstructive pulmonary disease and heart valve disease in his history. During the physical examination on admission his body temperature was $38.2^{\circ} \mathrm{C}$. Diastolic murmur of grade $3 / 6$ at aortic area, and systolic murmur grade of $2 / 6$ in mitral area were found. Brucella agglutination tests (Rose-Bengal and Wright) were studied in serum sample. Both Rose-Bengal and Wright tests (the latter in a titer of 1/ 1,280 ) were positive. In transthoracic echocardiography examination, active vegetation was present on aortic valve with the size of $1 \times 1.4 \mathrm{~cm}$. A heart valve operation was planned-following a 6-week antibiotic therapy with rifampicin and doxycycline with a combination of streptomycin for 3 weeks. The patient, who developed respiratory and cardiac arrest on the 5th day of admittance, did not give response to resuscitation and died. After 2 days, Brucella 


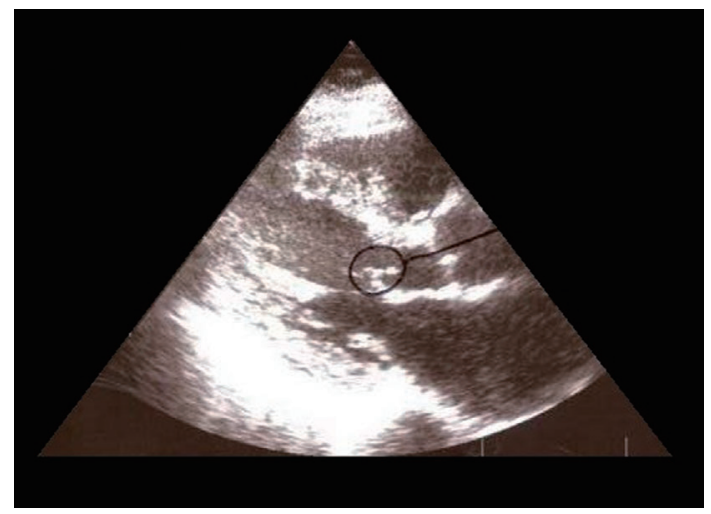

Figure 1. The transthoracic echocardiography showing vegetation on the atrial side of the anterior mitral leaflet (the circle shows the vegetation).

melitensis was isolated from blood culture drawn on admission.

\section{Case 2}

A 54-year-old woman visited our hospital for Brucella examination upon diagnosis of her husband with Brucella epididymo-orchitis. The patient, who had no characteristic symptoms in her history, had temperature: $36.8^{\circ} \mathrm{C}$, pulse: $80 /$ min, arterial blood pressure: $90 / \mathrm{mmHg}$ and systolic murmur of grade $2 / 6$ at mitral area in the examination. Other system examinations were normal.

TTE showed $1.0 \times 1.1 \mathrm{~cm}$ vegetation on the atrial side of the anterior mitral leaflet, consistent with infective endocarditis (Fig. 1). In laboratory examinations, the Brucella Wright titer was found to be $1 / 640$. A vegetative formation on the mitral valve was seen in echocardiography examination. Rifampicin, doxycycline, and streptomycin treatments were given to the patient with diagnosis of Brucella endocarditis. B. melitensis was recovered from successive blood cultures and bone marrow aspiration culture. No valve operation was considered for the patient by cardiovascular surgery department. Nine-month medical treatment was planned for the patient. Streptomycin was ceased after 3 weeks and trimetoprim-sulphamethoxazole treatment was initiated. The patient is in the 8th month of treatment and her disease is still under control without any problems.

\section{Discussion}

Endocarditis is the most frequent cause of mortality in brucellosis. Endocarditis was found in $80 \%$ of fatal brucellosis cases (5). Sasmazel et al. evaluated 186 patients with a diagnosis of endocarditis; Brucella endocarditis was found in $13(7 \%)$ of them (6). Different from endocarditis related to other bacteria, the Brucella endocarditis has tendency of fibrosis, hyalinization and calcification. Mortality in Brucella endocarditis is mostly related with heart failure. In $82 \%$ of the cases, aortic valve involvement was reported (7). Mitral valve involvement is less frequent.

Development of cardiac failure, hemodynamic failure, big vegetation, existence of emboli, valvula damage, and abscess formation are the main surgical indications in brucella endocarditis (8). Erbay et al. presented a case of Brucella melitensis endocarditis complicated with a superior mesenteric artery aneurysm. In that case, since there was no intracardiac complication of Brucella endocarditis, and with medical therapy a significant reduction in the size of the vegetation on the mitral valve leaflets was noticed, they did not perform any cardiac surgery such as mitral valve repair or replacement (9). Medical therapy can be used for patients with mild cardiac involvement and with symptoms of short duration $(10,11)$. In the present second case, the patient is being treated only with medical therapy for those reasons. To our knowledge only 16 cases have been reported in the literature who have been cured with medical therapy alone (12).

In the present second case, the patient was diagnosed even though there were no symptoms. She was tested for brucellosis because her husband had been diagnosed with Brucella epididimoorchitis. Serological screening of family members is generally suggested in endemic areas (13).

In conclusion, Brucella spp. should be considered as a causative organism among patients with endocarditis. And rare complications like endocarditis are possible in patients diagnosed with Brucella infection, as the brucellosis is endemic in Turkey. Medical therapy alone can be considered for some chosen patients with Brucella endocarditis. We think that the initial clinical presentation of disease is one of the major predictive factors even though optimum treatment and prognostic criteria are not well defined for Brucella endocarditis.

The authors state that they have no Conflict of Interest (COI).

\section{References}

1. Mert A, Kocak F, Ozaras R, et al. The role of antibiotic treatment alone for the management of Brucella endocarditis in adults: a case report and literature review. Ann Thorac Cardiovasc Surg 8: 381-385, 2002.

2. Cay S, Cagirci G, Maden O, Balbay Y, Aydogdu S. Brucella endocarditis - a registry study. Kardiol Pol 67: 274-280, 2009.

3. Jeroudi MO, Halim MA, Harder EJ, Al-Siba'i MB, Ziady G, Mercer EN. Brucella endocarditis. Br Heart J 58: 279-283, 1987.

4. Gould FK, Denning DW, Elliott TS, et al. Guidelines for the diagnosis and antibiotic treatment of endocarditis in adults: a report of the Working Party of the British Society for Antimicrobial Chemotherapy. J Antimicrob Chemother 2011.

5. Peery TM, Belter LF. Brucellosis and heart disease. II. Fatal brucellosis: a review of the literature and report of new cases. Am J Pathol 36: 673-697, 1960.

6. Sasmazel A, Baysal A, Fedakar A, et al. Treatment of Brucella endocarditis: 15 years of clinical and surgical experience. Ann Thorac Surg 89: 1432-1436, 2010.

7. Reguera JM, Alarcon A, Miralles F, Pachon J, Juarez C, Colmenero JD. Brucella endocarditis: clinical, diagnostic, and therapeutic approach. Eur J Clin Microbiol Infect Dis 22: 647-650, 2003.

8. Tuncer M, Ekim H, Gunes Y, Guntekin U. Atrial septal defect pre- 
senting with Brucella endocarditis. Circ J 72: 2096-2097, 2008.

9. Erbay AR, Turhan H, Dogan M, Erbasi S, Cagli K, Sabah I. Brucella endocarditis complicated with a mycotic aneurysm of the superior mesenteric artery: a case report. Int J Cardiol 93: 317-319, 2004.

10. Ferreira P, Gama P, Correia J, et al. Brucella endocarditis--case report and literature review. Rev Port Cardiol 27: 1309-1315, 2008.

11. Purwar S, Metgud SC, Darshan A, Mutnal MB, Nagmoti MB. In- fective endocarditis due to brucella. Indian J Med Microbiol 24: 286-288, 2006.

12. Mohandas N, Balasubramanian R, Prasad SB. Can brucella endocarditis be treated successfully with medical therapy alone? Trop Doct 39: 123-124, 2009.

13. Almuneef MA, Memish ZA, Balkhy $\mathrm{HH}$, et al. Importance of screening household members of acute brucellosis cases in endemic areas. Epidemiol Infect 132: 533-540, 2004.

(C) 2012 The Japanese Society of Internal Medicine http://www.naika.or.jp/imindex.html 